\title{
Estimation of Durability of Profit of Small and Medium Enterprises by Statistical Matching
}

\author{
Yukiko KURIHARA \\ Faculty of Humanities, Hirosaki University, Japan.
}

Received: February 27, 2015 / Accepted: March 25, 2015 / Published: May 25, 2015.

\begin{abstract}
This study computes the durability of Return on Assets (ROA) in small and medium enterprises from different sample datasets. Utilizing information from the Financial Statements Statistics of Corporations by Industry, it verifies the precision of correlation coefficients using the Non-iterative Bayesian-based Imputation (NIBAS) and multiple imputation method for all combinations of common variables with auxiliary files. The following are the three important findings of this paper. First, statistical matching estimates of higher precision can be obtained using key variable sets with higher canonical correlation coefficients. Second, even if the key variable sets have high canonical correlation coefficients, key variables that are correlated extremely strongly with target variables and have high kurtosis should not be used. Finally, using auxiliary files can improve the precision of statistical matching estimates. Accordingly, the durability of ROA in small and medium enterprises is computed. The author finds that the series of ROA correlation fluctuates for smaller enterprises compared to larger ones, and thus, the vulnerability of ROA in small and medium enterprises can be clarified via statistical matching.
\end{abstract}

Keywords: Bayesian regression imputation, Multiple imputation, Canonical correlation coefficient, Sampling experiment.

\section{Introduction}

In order to observe the durability of Return on Assets (ROA), the autocorrelation between outputs at periods $t$ and $t-4$ can be computed using panel data and exact matching with the identifier. Using the quarterly survey data from the Financial Statements Statistics of Corporations by Industry (FSS) (Ministry of Finance, Japan), Figure 1 shows the results for two-year panel data with the identifier. Large enterprises are classified under Group 1 (capital of over 1 billion yen; indicated by the black line) or Group 2 (capital of 100 million to 1 billion yen; indicated by the dashed line).

From FY1984 to 2011, the autocorrelation of ROA fluctuated moderately. However, there were two downturns because of the depression and the Lehman collapse. Group 2 displays a similar pattern as Group

Corresponding author: Yukiko KURIHARA, Ph.D., research field: economic statistics, complex survey methods. E-mail: yukuri@hirosaki-u.ac.jp.
1, albeit with larger fluctuations than those in Group 1. This suggests that smaller enterprises are more vulnerable.

As the FSS surveys large enterprises with capital stock of over one billion yen, panel data can be created for large enterprises alone by means of exact matching with the identifier. However, small and medium enterprises are randomly sampled and surveyed for only one year (across four quarterly periods). Thus, the data for small and medium enterprises cannot be linked or analyzed across different survey years. If the conditions required for statistical matching for constructing short-term panel data are fulfilled, short-term panel analysis could be conducted for enterprises of all sizes.

Depending on the linkages between existing data, statistical matching enables comprehensive utilization of individual data resources and micro-data analyses into new frameworks without conducting additional surveys and expending more resources. However, estimates from matching data contain 
ineluctable errors caused by the uncertainty of statistical matching; different individuals across several data resources are interconnected based on variables commonly included in different data resources. These variables are called common variables or key variables.

Rässler [6] conducted a pioneering study on statistical matching by comparing several parametric matching models and verified the features of estimates from these models. Araki and Yoshizoe [1] investigated the differences between the constrained and unconstrained distance hot-deck matching technique, using linkages between the Survey of Household Economy and the Family Savings Survey (Statistics Bureau of Japan). Kurihara [3] attempted an analysis of pseudo-panel data created by the Mahalanobis distance technique by utilizing the Survey of Small and Medium Enterprise Agency Business Conditions (Ministry of Economy, Trade and Industry). Sakata and Kurihara [7] applied nonparametric and parametric matching methods to FSS data (Ministry of Finance) and analyzed the biases and mean squared errors of estimates from matching data.

Thus, previous works have primarily contributed to the specification of useful methods through comparisons between statistical matching methods (i.e., parametric and non-parametric). However, the error of statistical matching is not only influenced by the matching technique itself but also by the available common variables in different data sets and their distributions. Specifically, when we have only separate data sources, true values are unknown, and estimates on complete datasets are unavailable. Hence, it is difficult to evaluate the precision of statistical estimates from matching data.

The following procedure is generally adopted to verify the effectiveness of statistical matching. i) Prepare similar datasets that can be completely or partially linked by identifiers, ii) clarify distributions and peculiarities among estimates from matching data by comparing estimates from complete data of sampling experiments, and finally, iii) apply these results to actual / separate datasets.

Therefore, the aim of this paper is to verify the concrete effects of the selection of common variables as well as the effectiveness of auxiliary files, to create a panel dataset of FSS. Based on these results, small and medium enterprises appearing in different sample datasets are linked by statistical matching, and the durability of ROA is computed.

\section{Overview of Statistical Matching}

The correlation coefficients of variables $X$ and $Y$ are set as the target estimates; hence, variables $X$ and $Y$ are called target variables. We cannot obtain a resource that contains both $X$ and $Y$, but we can use dataset A, with $Y$, and dataset $\mathrm{B}$, with $X$, separately.

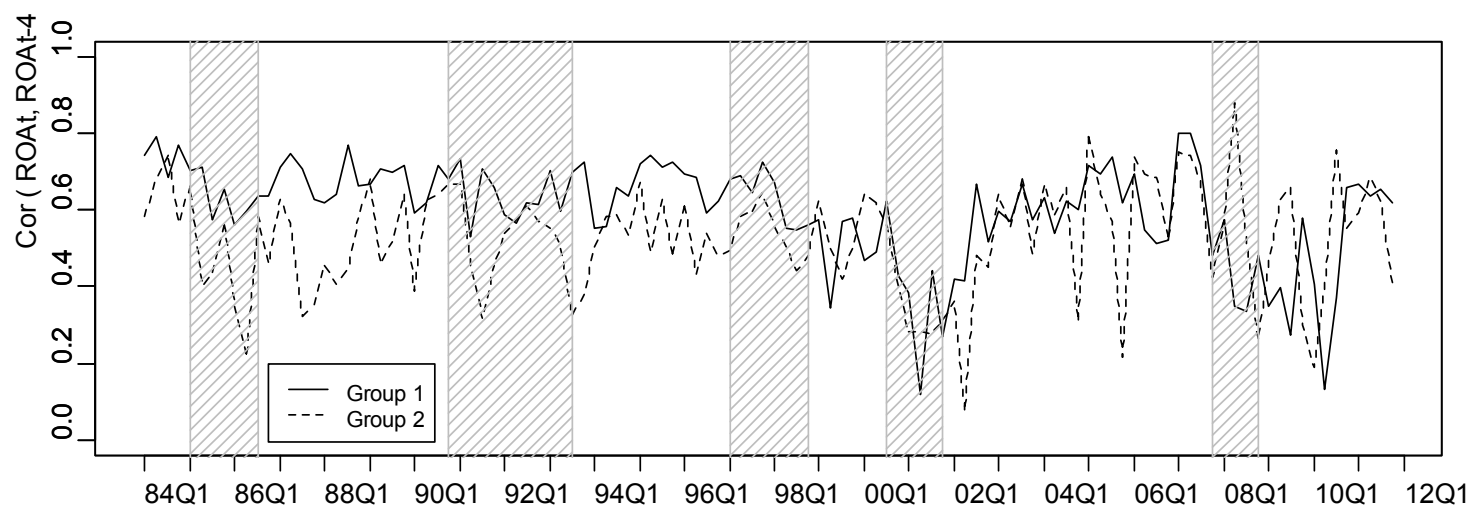

Fig. $1 \operatorname{Cor}\left(\mathrm{ROA}_{t}, \mathrm{ROA}_{t-4}\right)$ of large enterprises in the electric machinery manufacturing sector.

Note: Group 1 is enterprises with a capital of over 1 billion yen and indicated by the black line, and also Group 2 is enterprises with a capital of 100 million to 1 billion yen and indicated by the dashed line. 
Datasets $\mathrm{A}$ and $\mathrm{B}$ share a common variable, $Z$, called the key variable. That is, dataset $\mathrm{A}[Y, Z]$ and dataset $\mathrm{B}$ $[X, Z]$.

When dataset A corresponds to the recipient file, which contains basic information, and dataset B corresponds to the donor file, which includes additional variables, statistical matching creates extension data $[X, Y, Z]$ by adding $X$ to dataset $\mathrm{A}[Y, Z]$ based on key variable $Z$. The following section outlines the theoretical underpinnings of statistical matching.

\subsection{Methodology}

Statistical matching methods are categorized as non-parametric and parametric. Non-parametric methods use distance functions for key variables, search for individuals that satisfy the nearest distance between dataset $\mathrm{A}$ and $\mathrm{B}$, and then link individuals. Several distances, such as the constrained distance, unconstrained distance, Mahalanobis distance, and so on, are used for matching. ${ }^{1}$

Parametric methods apply statistical models to key variables and target variables, estimate parameters of theoretical distributions, and then impute new values that are generated stochastically from estimated distributions. Several models based on imputation methods have been developed for parametric matching methods (e.g., Bayesian regression imputation and data augmentation).

The present study uses Non-iterative Bayesian-based Imputation (NIBAS), a parametric method, for matching as it provides more precise values. ${ }^{2}$ NIBAS assumes a multivariate normal distribution, derives parameters $\mu_{X \mid Z Y}, \mu_{Y \mid Z X}, \Sigma_{X \mid Z Y}$, and $\Sigma_{Y \mid Z X}$ from regression models, and stochastically generates values for target variables using equations (1) and (2).

$$
X \mid y, \beta, \Sigma \sim N\left(\mu_{X \mid Z Y} ; \Sigma_{X \mid Z Y}\right),
$$

\footnotetext{
${ }^{1}$ See D’Orazio, Di Zio and Scanu [2] for details about non-parametric matching.

${ }^{2}$ See Rässler [7; pp. 96-107] for theoretical details about regression imputation methods, including NIBAS. Imputation methods is elaborated in Little and Rubin [4].
}

$$
Y \mid x, \beta, \Sigma \sim N\left(\mu_{Y \mid Z X} ; \Sigma_{Y \mid Z X}\right),
$$

Vectors $X, Y$, and $Z$ consist of variables of sizes $q, p$, and $h$, respectively. $\beta_{X Z}$ and $\beta_{Y Z}$ denote parameter estimates from the regression of $X$ on $Z_{B}$ and the regression of $Y$ on $Z_{A}$, respectively. Also, $\Sigma_{X X \mid Z}$ (or $\Sigma_{Y Y \mid Z}$ ) denotes the variance-covariance matrix of $X$ (or $Y$ ) given Z. $\hat{\Sigma}_{Y X \mid Z}\left(\operatorname{or} \hat{\Sigma}_{X Y \mid Z}\right)$ is the conditional covariance of $X$ and $Y$ given $Z$.

$$
\begin{gathered}
\mu_{X \mid Z Y}=Z_{A} \beta_{X Z}+\left(Y-Z_{A} \beta_{Y Z}\right) \Sigma_{Y Y \mid Z}^{-1} \hat{\Sigma}_{Y X \mid Z} \\
\mu_{Y \mid Z X}=Z_{B} \beta_{Y Z}+\left(X-Z_{B} \beta_{X Z}\right) \Sigma_{X X \mid Z}^{-1} \hat{\Sigma}_{X Y \mid Z} \\
\Sigma_{X \mid Z Y}=\left(\Sigma_{X X \mid Z}-\hat{\Sigma}_{X Y \mid Z} \Sigma_{Y Y \mid Z}^{-1} \hat{\Sigma}_{Y X \mid Z}\right) \otimes I_{n a} \\
\Sigma_{Y \mid Z X}=\left(\Sigma_{Y Y \mid Z}-\hat{\Sigma}_{Y X \mid Z} \Sigma_{X X \mid Z}^{-1} \hat{\Sigma}_{X Y \mid Z}\right) \otimes I_{n b}
\end{gathered}
$$

Furthermore, we use stochastically generated values from the inverse Wishart distribution as estimates of $\Sigma_{X X \mid Z}$ and $\Sigma_{Y Y \mid Z}$. Also, $\beta_{X Z}$ (or $\beta_{Y Z}$ ) are stochastically generated from the normal distribution with mean $\hat{\beta}_{X Z}$ (or $\hat{\beta}_{Y Z}$ ) and the variance that consists of the Kronecker product of $\hat{\Sigma}_{X X \mid Z}$ (or $\hat{\Sigma}_{Y Y \mid Z}$ ) and the inverse of $Z_{B}^{\prime} Z_{B}$ (or $Z_{A}^{\prime} Z_{A}$ ). These generated values alter for each matching time.

As $\hat{\Sigma}_{Y X \mid Z}$ and $\hat{\Sigma}_{X Y \mid Z}$ can only be computed from the complete data $[X, Y, Z]$, they are unknown when using incomplete data. If we have auxiliary files about $[X, Y, Z]$ (e.g., complete data from a small sample), we can calculate this covariance matrix. However, if there is no additional information, we must insert zeroes for both $\hat{\Sigma}_{Y X \mid Z}$ and $\hat{\Sigma}_{X Y \mid Z}$.

There is a risk of overstepping the range of the actual data because imputation values are generated stochastically based on the theoretical distribution. To resolve this problem, the truncated multivariate normal distribution with maximums and minimums of datasets is applied.

The imputation values fluctuate between matching instances, so that the multiple imputation method, wherein the estimation (MI estimates) is the average of several matching values, needs to be utilized. Multiple imputation assumes these fluctuations arise from the uncertainty of statistical matching, and it evaluates the standard error to account for the 
uncertainty. $^{3}$

\subsection{Conditions for Statistical Matching}

Generally, statistical matching methods with key variables $Z$ assume that the Conditional Independence Assumption (CIA) is true. This means that distributions $X$ and $Y$, given $Z$, are independent.

$$
f(X, Y \mid Z)=f(X \mid Z) f(Y \mid Z)
$$

As complete data are required for confirming the CIA, we cannot check the CIA in the actual analysis of statistical matching using two separate resources.

The main available resources for improving precision are the key variables $Z$, and these should be selected on the condition that target variables strongly correlate with key variables. Therefore, the primary condition of key variables selection is attaining high precision estimation by statistical matching. The Canonical Correlation Coefficient Estimation (CCE) is useful for measuring the correlation between key variables and target variables. CCE helps obtain the correlation between two groups by computing the component indicator of the correlation structure between several variables.

\section{Data and Matching Procedure}

\subsection{Data}

As the FSS surveys all large enterprises, panel data can be created only among large enterprises by means of exact matching with the identifier. Thus, the selection effect of key variables could be proved by comparing the results from matching panel data and complete panel data. Quarterly data from FSS is used as the dataset for measuring the selection effect of key variables. The target sample ${ }^{4}$ includes enterprises in Group 2, namely large enterprises with a capital of

\footnotetext{
${ }^{3}$ The $\mathrm{R}$ program for statistical matching and multiple imputation was developed by the author after referring to the SPLUS code in Rässler (6; pp. 214-221) and the basic programming techniques of $\mathrm{R}$ in Mase [5].

${ }^{4}$ Outliers are excluded on the base of Mahalanobis distance at the significance level of 0.01 .
}

100 million to 1 billion yen in the manufacturing sector. Different survey periods can be connected using the identifier.

The target estimate is the correlation coefficient between the ROAs at survey periods $t$ (FY1984Q1) and $t-4$ (FY1985Q1), specifying the condition of key variables. Then, the target variables $X$ in the recipient file and $Y$ in the donor file are set to the ROA at periods $t$ and $t-4$. Moreover, $Z 1$ to $Z 5$ denote key variables, as noted in Table $1 .^{5}$ In order to test the combination effects of the key variables, five variables having different strengths of correlation with the target variables are chosen. Furthermore, the effects of the auxiliary file for matching are verified using data from Group 1 (i.e., large enterprises with a capital of 1 billion yen or more).

The distributions and Q-Q plot of variables are presented in Figure 2. Most variables hardly satisfy the normality assumption. The correlation coefficients between target variables and key variables (Table 3) indicate that the key variables Z1 and Z2 strongly correlate with $\mathrm{X}$ or $\mathrm{Y}$; however, the kurtosis is not close to zero (Table 2).

\subsection{Matching Process}

The process for verifying the selection effect of key variables is conducted as follows (Figure 3).

1) Let datasets $A$ and $B$ link each individual enterprise by exact matching through the identifier as the population dataset, and compute the true value $\theta_{a}$.

2) Conduct sampling stochastically, with size $n_{1}=100$, from the population data, where completely different samples are drawn from A and B. Sampling data for $\mathrm{A}$ and $\mathrm{B}$ are represented by $\mathrm{A}^{X \mathrm{mis}}$ and $\mathrm{B}^{\text {Ymis }}$, respectively, because data $\mathrm{A}^{X \text { mis }}$ do not include $X$ and $\mathrm{B}^{Y \text { mis }}$ do not include $Y$.

\footnotetext{
5 Statistical matching with parametric methods basically targets the estimation of joint distributions in relation to key variables. If we estimate parameters with transformed values, such as ratios from matching data, we can obtain estimates of higher precision by matching the transformed data than by matching the original data directly and subsequently transforming the matching data.
} 
Table 1 Dataset for Statistical Matching.

\begin{tabular}{lll}
\hline & Data A : period $t$ & Data B : period $t-4$ \\
\hline$X$ & Missing & Ratios of operating profits to total assets \\
$Y$ & Ratios of operating profits to total assets & Missing \\
$Z 1$ & Ratios of ordinary profits to total assets & Ratios of ordinary profits to total assets \\
$Z 2$ & Ratios of operating profits to sales & Ratios of operating profits to sales \\
$Z 3$ & Salaries for employees & Salaries for employees \\
$Z 4$ & Return on equity & Return on equity \\
$Z 5$ & Total assets turnover & Total assets turnover \\
\hline
\end{tabular}

Table 2 Kurtoses and Skewness of Group 2 (FY1985Q1).

\begin{tabular}{lllllll}
\hline & X $(Y)$ & Z1 & Z2 & Z3 & Z4 & Z5 \\
\hline Kurtosis FY1984Q1 & 2.217 & 2.015 & 3.174 & 6.069 & 4.860 & 2.898 \\
Kurtosis FY1985Q1 & 3.384 & 3.420 & 6.363 & 10.516 & 4.967 & 2.860 \\
Skewness FY1984Q1 & 0.481 & 0.565 & -0.194 & 2.066 & -0.614 & 1.231 \\
Skewness FY1985Q1 & 0.443 & 0.598 & -0.438 & 2.618 & -0.559 & 1.286 \\
\hline
\end{tabular}

Table 3 Correlation between key variables and target variables of Group 2 (FY1985Q1).

\begin{tabular}{llllll}
\hline & Z1 & Z2 & Z3 & Z4 & Z5 \\
\hline X (FY1984Q1) & 0.963 & 0.883 & 0.060 & 0.360 & 0.219 \\
Y (FY1985Q1) & 0.964 & 0.859 & 0.109 & 0.365 & 0.184 \\
\hline
\end{tabular}
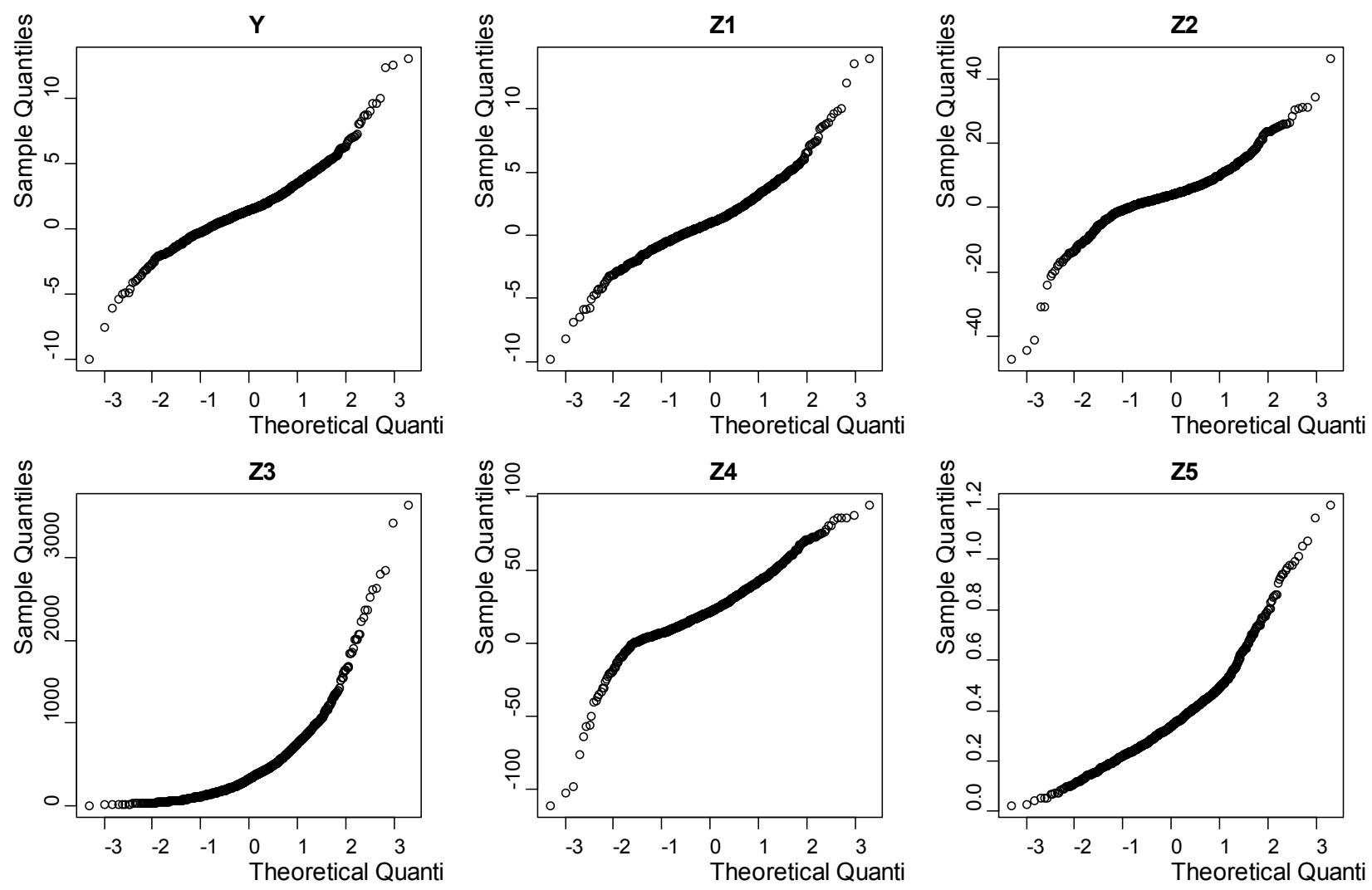

Fig. 2 Q-Q plot (FY1985Q1, Group 2). 


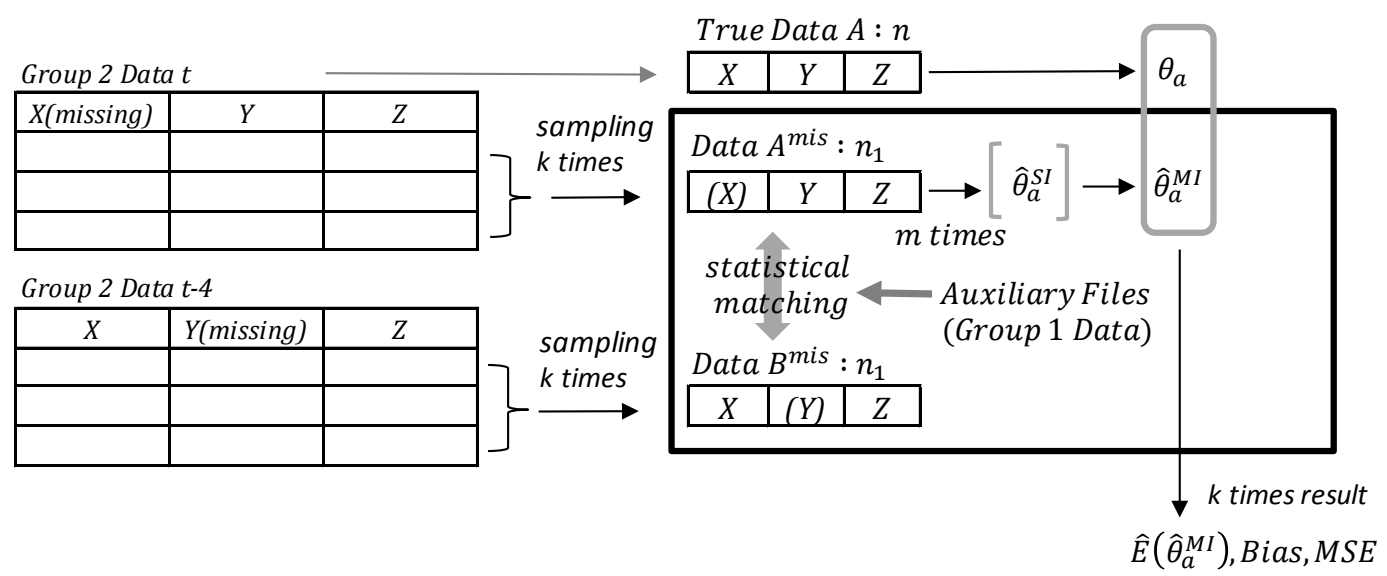

Fig. 3 Process of the statistical matching.

3) Create data $[X, Y, Z]$ by connecting individuals of $\mathrm{B}^{Y \mathrm{mis}}$ to individuals of $\mathrm{A}^{X \mathrm{mis}}$.

4) Compute the estimated value of the correlation coefficient with the matching data. The estimate computed by only one matching trial is called the single imputation estimate $\left(\hat{\theta}_{a}^{S I}\right)$.

5) Repeat steps (3) and (4) $M=30$ times, collect estimates, and then compute multiple imputation estimates $\hat{\theta}_{a}^{M I}$.

6) For measuring the difference between samples, repeat steps (2) through (5) $K=100$ times, and calculate expectation estimates of multiple imputation estimates $\hat{E}\left(\hat{\theta}_{a}^{M I}\right)$, bias, and mean squared errors (MSEs).

$$
\begin{gathered}
\hat{E}\left(\hat{\theta}_{a}^{M I}\right)=\frac{\sum_{k=1}^{K} \widehat{\theta}_{a, k}^{M I}}{K} \\
\text { Bias }=\hat{E}\left(\hat{\theta}_{a}^{M I}\right)-\theta_{a} \\
M S E=\frac{\sum_{k=1}^{K}\left(\widehat{\theta}_{a}^{M I}-\theta_{a}\right)^{2}}{K}
\end{gathered}
$$

7) Repeat steps (2) through (6), altering combinations of key variables (31 combinations in all), and specify the relation between the combinations of key variables (strength of CCE) and biases or MSEs. Apply the results to estimate the correlation coefficient in Group 3 , which consists of different samples.

\section{Results}

\subsection{Matching Results by Combinations of Key Variables Without Auxiliary Files}

The selection of key variables determines the precision of the estimates based on matching data. In order to ascertain the effects of their combinations, matching experiments are conducted by altering the combinations of key variables. In this case, there are 31 possible combinations in all among the five key variables in the first quarter of 1985. Figure 4 shows the biases or MSEs of the MI estimates and the CCEs on the vertical and horizontal axes, respectively. Groups A, B, and C denote the results by the key variable set including $Z 1$, by the key variable set including $Z 2$ and excluding $Z 1$, and by the key variable set excluding both $Z 1$ and $Z 2$, respectively.

Biases and MSEs are low if key variable sets, except for Group A, with higher CCEs are utilized. Even if the key variable set indicates an extremely strong CCE for Group A (which includes Z1 key variables), the biases and MSEs in this case are large. This is because $Z 1$ and the target variables have an extremely strong correlation (almost equal to one), and the kurtosis is high; a key variable that does not follow the normal distribution but has an extremely strong relation with target variables increases the bias or MSE.

Table 4 indicates the best five combinations of key variables ranked by bias. The best five combinations include $Z 2$ and exclude $Z 1$. Therefore, $Z 2$ is a very effective key variable, while $Z 1$ is an ineffective key variable. Therefore, the selection condition specifies including any key variables, except $Z 1$, with higher CCE. 


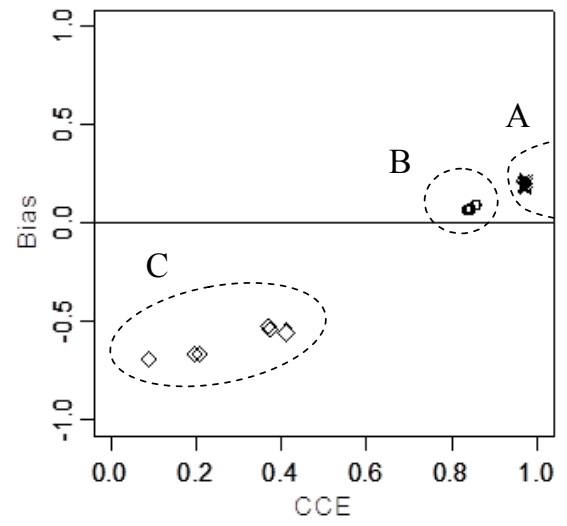

(a) Bias and CCE

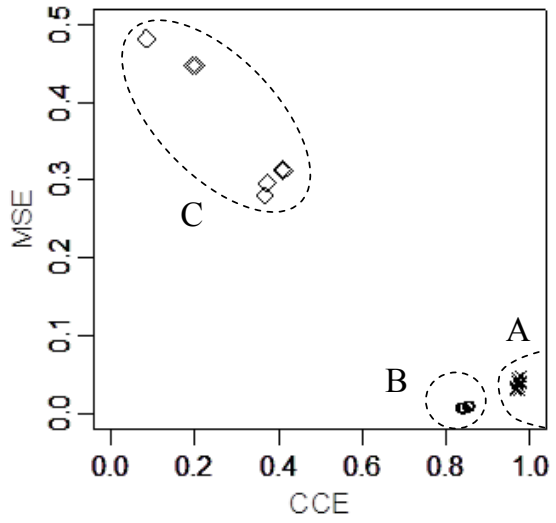

(b) MSE and CCE

Fig. 4 Relation between CCE and bias or MSE depending on combinations of key variables (FY1985Q1).

Note: Group A $(\times)$ comprises the variable set with $Z 1$; Group B (०), with $Z 2$ and without $Z 1$; and Group C $(\diamond)$ without both $Z 1$ and $Z 2$.

Table 4 Combinations of Key Variables, Bias, MSE, and CCE by Bias Ranking.

\begin{tabular}{|c|c|c|c|c|c|c|c|c|}
\hline \multirow{2}{*}{ Bias ranking } & \multicolumn{5}{|c|}{ Combinations } & \multirow{2}{*}{-Bias } & \multirow{2}{*}{ MSE } & \multirow{2}{*}{$\mathrm{CCE}$} \\
\hline & $Z 1$ & $Z 2$ & $Z 3$ & $Z 4$ & $Z 5$ & & & \\
\hline Best & 0 & 1 & 0 & 0 & 0 & 0.0599 & 0.00617 & 0.8361 \\
\hline 2 nd & 0 & 1 & 1 & 0 & 0 & 0.0602 & 0.00610 & 0.8391 \\
\hline $3 \mathrm{rd}$ & 0 & 1 & 0 & 1 & 0 & 0.0611 & 0.00616 & 0.8410 \\
\hline 4 th & 0 & 1 & 1 & 1 & 0 & 0.0660 & 0.00665 & 0.8434 \\
\hline 5 th & 0 & 1 & 1 & 1 & 1 & 0.0823 & 0.00902 & 0.8587 \\
\hline
\end{tabular}

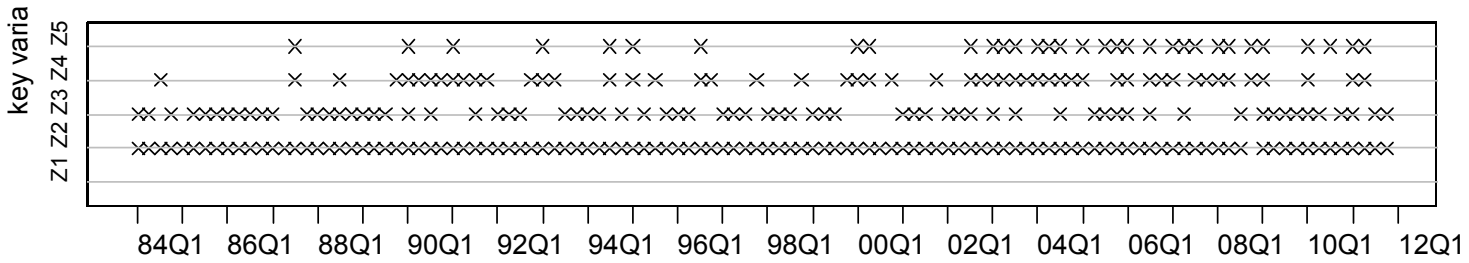

Fig. 5 Variable sets selected according to the specified condition.

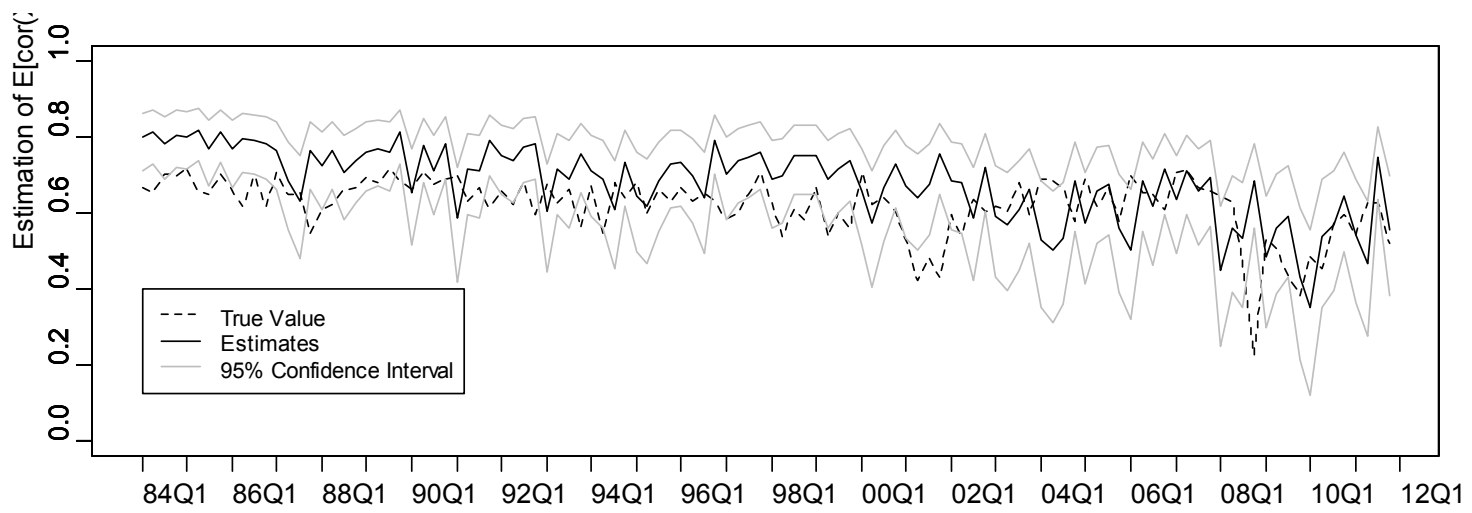

Fig. 6 Matching results of ROA durability for enterprises in the manufacturing sector. 
According to the verified condition, the key variable set is selected (Figure 5), and the sets differ by survey periods. The matching results are computed in Figure 6, and estimates obviously have large biases from the true ROA autocorrelations. Thus, auxiliary information must be utilized for improving precision.

\subsection{Matching Results by Combinations of Key Variables with Auxiliary Files}

Figure 7 depicts the relation between CCEs and biases or MSEs, utilizing the Group 1 file as the auxiliary file. Regarding Groups A and B, which include either $Z 1$ or $Z 2$, the matching estimations have large biases and MSEs even if the CCE is high. The five best results include only Z3, Z4, and Z5 in Table 5. The reasons $Z 1$ and $Z 2$ do not contribute toward improving the precision of matching estimates include the strong correlation with target variables and the high kurtosis. In order to obtain matching results with high precision when the auxiliary file is used, the key variable sets must have high CCE and exclude $Z 1$ and Z2.

Based on these results, $[Z 3, Z 4, Z 5]$ are selected as the best combinations of key variable sets over all survey periods. The matching results with the auxiliary file by survey periods are computed in Figure 8. Results with an auxiliary file show improved precision and the $95 \%$ confidence interval includes the true values.

\subsection{Results for Small and Medium Enterprises}

The results of the sampling experiments for Group 2 indicate that the best key variable sets for matching with Group 3 (small and medium enterprises in the electric machinery manufacturing sector) are $[Z 3$, $Z 4, Z 5]$ through all survey periods. Thus, the durability of ROA in these small and medium enterprises is estimated by statistical matching, utilizing data from

Table 5 Combinations of Key Variables, Bias, MSE, and CCE by Bias Ranking.

\begin{tabular}{llllllll}
\hline \multirow{2}{*}{ Bias ranking } & \multicolumn{9}{c}{ Combinations } & & MSE \\
\cline { 2 - 7 } & $Z 1$ & $Z 2$ & $Z 3$ & $Z 4$ & $Z 5$ & Bias & 0.00131 \\
\hline Best & 0 & 0 & 0 & 1 & 1 & -0.0200 & 0.4108 \\
2nd & 0 & 0 & 0 & 0 & 1 & -0.0319 & 0.00156 \\
3rd & 0 & 0 & 1 & 1 & 1 & -0.0379 & 0.00242 \\
4th & 0 & 0 & 1 & 0 & 1 & -0.0447 & 0.00262 \\
5th & 0 & 0 & 0 & 1 & 0 & -0.0482 & 0.4120 \\
\hline
\end{tabular}

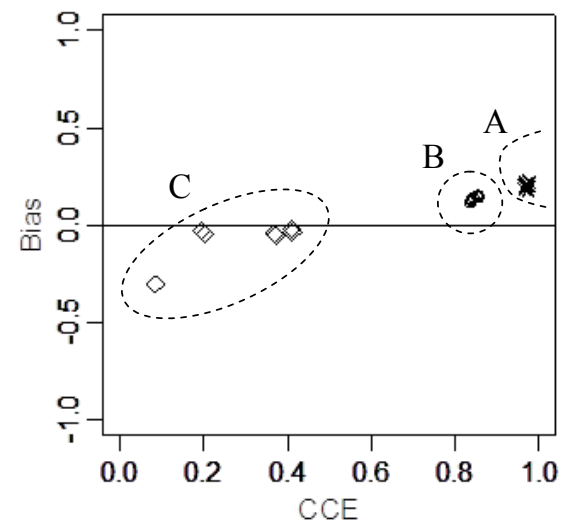

(a) Bias and CCE

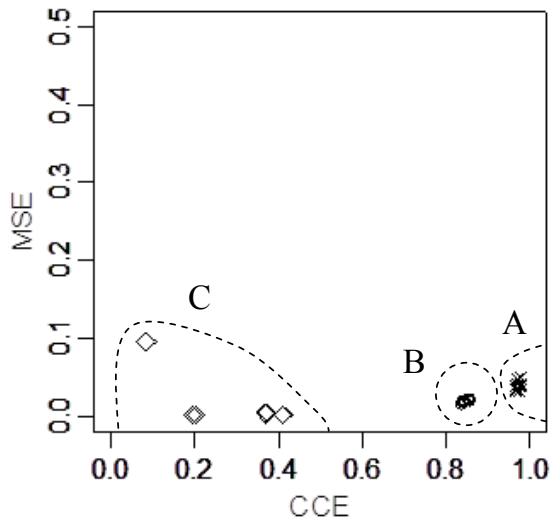

(b) MSE and CCE

Fig. 7 Relation between CCE and bias or MSE by combinations of key variables with the auxiliary file (FY1985Q1).

Note: Group A $(\times)$ denotes the variable set with $Z 1$, Group B (०), with $Z 2$ and without $Z 1$; and Group C $(\diamond)$, without both $Z 1$ and Z2. 


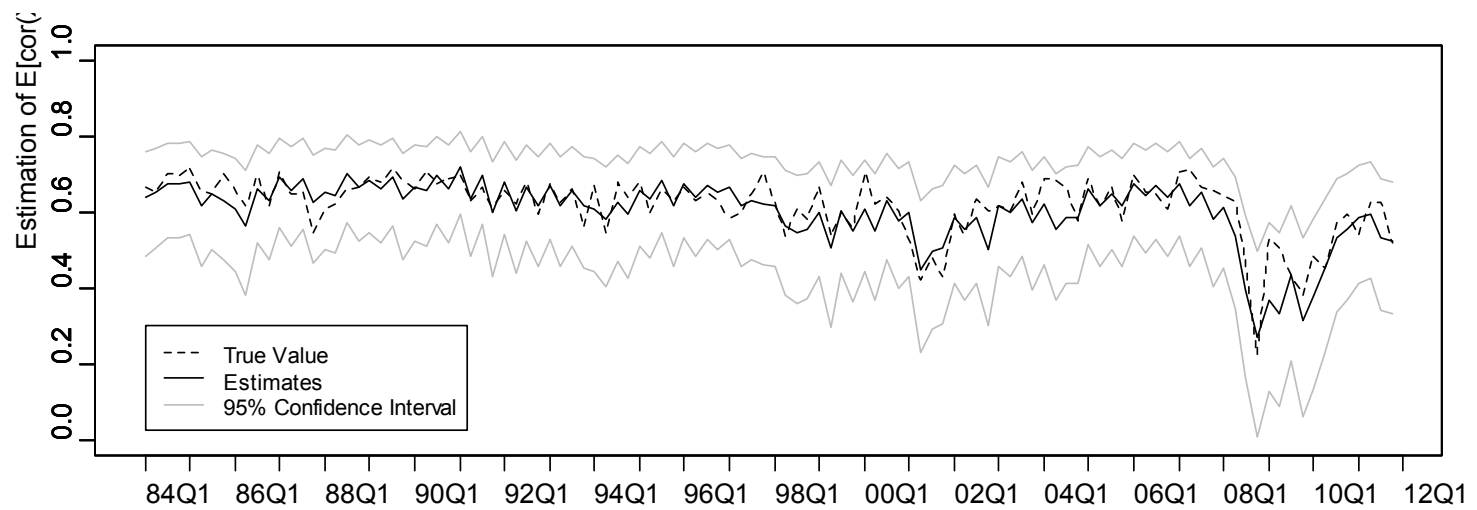

Fig. 8 Matching results of ROA durability with auxiliary files for enterprises in the manufacturing sector.

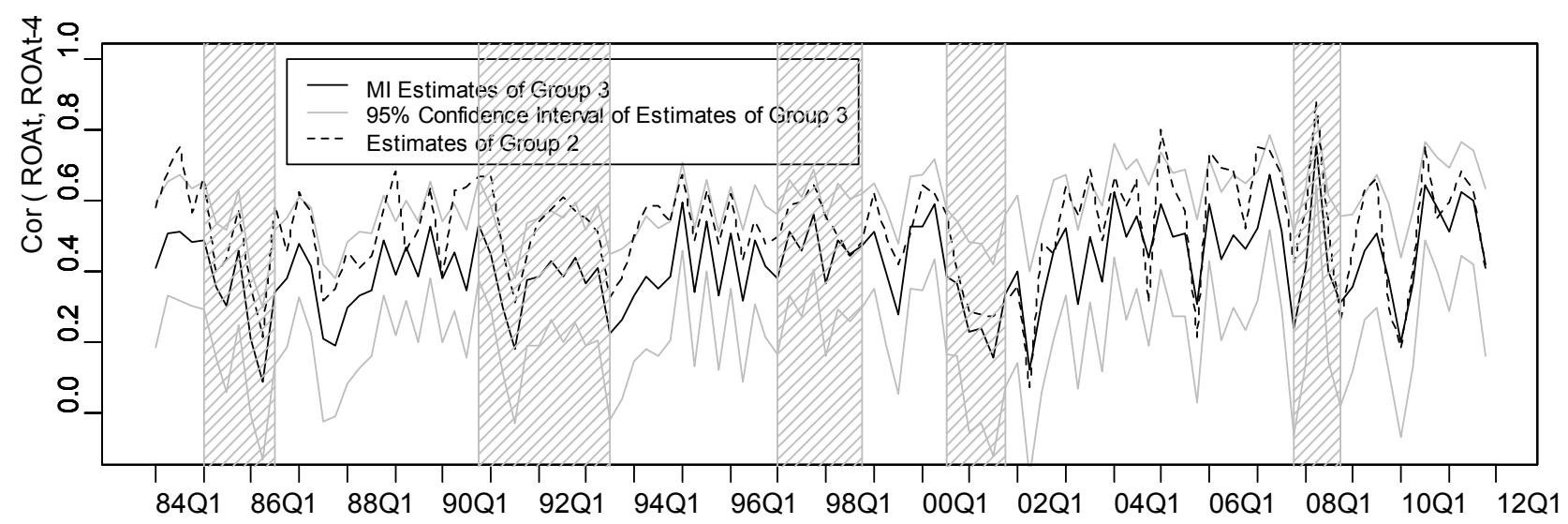

Fig. $9 \operatorname{Cor}\left(\mathrm{ROA}_{t}, \mathrm{ROA}_{t-4}\right)$ of small and medium enterprises in the electric machinery manufacturing sector.

Note: Group 2 is enterprises with a capital of 100 million to 1 billion yen and indicated by the dashed line; Group 3 is enterprises with a capital of under 100 million yen and indicated by the black line.

Group 2 as the auxiliary information. The fluctuations through survey periods are similar to those of Group 2. However, the level of fluctuations is lower than that for large enterprises. Notably, statistical matching alone enabled us to verify the vulnerability of ROA in small and medium enterprises.

\section{Conclusion}

In order to construct the panel data using different sample datasets, this paper verified the precision of estimates using Bayesian-based statistical matching and multiple imputation for all combinations of common variables. Improvements in precision were also measured by utilizing different group files as auxiliary files. In conclusion, although CCEs may be high, the precision is poor if key variables are correlated extremely strongly with target variables and have large kurtosis. Therefore, these types of key variables are excluded, and key variable sets having high CCEs are optimal and should be selected.

A number of difficulties remain in applying statistical matching to real data, because these data rarely satisfy the normality, and selection methods for key variable sets have yet to be developed for non-normality situations. However, we cannot conduct a re-survey of past datasets, and statistical matching alone is expected to shed light on hitherto unobserved facts. Thus, the field of statistical matching needs more research and application across social statistics fields.

\section{Acknowledgments}

This work was supported by a research grant from the Joint Usage and Research Center, Institute of 
Economic Research, Hitotsubashi University. The analyses in this work were based on questionnaire information from the Quarterly Survey of Financial Statements Statistics of Corporations by Industry, from FY1983 to FY2012, provided by the Ministry of Finance. The author deeply appreciates the support of both the Ministry of Finance and the Research Centre for Information and Statistics of Social Science, Institute of Economic Research, Hitotsubashi University.

\section{References}

[1] M. Araki, Y. Yoshizoe, Exact matching and statistical matching using household survey data, Aoyama Journal of Business, 42(1) (2007) 175-209.

[2] M. D’Orazio, M. Di Zio, M.Scanu, Statistical matching: Theory and practice, Wiley, 2006.

[3] Y. Kurihara, Monte Carlo verification of correlation estimator precision based on statistical matching, The Annual Report of the Institute of Economics Research, Chuo University,43 (2012) 489-551.

[4] R.J.A. Little, D.B. Rubin, Statistical analysis with missing data, Wiley, 2002.

[5] S. Mase, R programming manual (in Japanese), Surikougaku-sha, 2010.

[6] S. Rässler, Statistical matching,Springer, 2002.

[7] Y. Sakata, Y. Kurihara, An empirical assessment of the validity of statistical matching on financial statements statistics, The Annual Report of the Institute of Economics Research, Chuo University, 44 (2013) 271-306 